\title{
Mehr Transparenz wagen!
}

\section{Konferenz der Informationsfreiheitsbeauftragten (IFK) in Deutschland, Kleinmachnow, 13.12.2010}

Die Informationsfreiheitsbeauftragten des Bundes und der Länder haben während ihrer Konferenz in Kleinmachnow die öffentlichen Stellen ausdrücklich aufgefordert, von sich aus die Initiative für mehr Transparenz zu ergreifen. In einer Entschließung zu Open Data erkennen sie an, dass viele Behörden inzwischen umfangreiche Informationen im Internet bereitstellen. Sie empfehlen, diese Aktivitäten zu bündeln und auf eine breitere Grundlage zu stellen. Plattformen in den USA und in Großbritannien zeigen, dass auch eine Vielzahl unterschiedlicher Informationen in einer für die Bürgerinnen und Bürger leicht zugänglichen Weise darstellbar ist. Die Vorsitzende der Konferenz - die Landesbeauftragte für den Datenschutz und für das Recht auf Akteneinsicht Brandenburg - Dagmar Hartge:

„Das Ziel einer transparenten Verwaltung darf kein Lippenbekenntnis bleiben. Je mehr staatliche Stellen Informationen aktiv bereitstellen, umso besser können die Bürgerinnen und Bürger ihre demokratischen Rechte wahrnehmen."

An die Gesetzgeber richtete die Konferenz den Appell, eine Rechtsgrundlage für die Veröffentlichung von Verträgen zwischen der öffentlichen Hand und privaten Unternehmen zu schaffen. Bislang muss ein Großteil dieser Daten wegen zu weit reichender gesetzlicher Ausnahmeklauseln geheim gehalten werden. Dagmar Hartge: "Aus Verträgen geht nicht nur die genaue Verwendung von Steuergeldern hervor, sie bestimmen in vielen Fällen auch weit in die Zukunft reichende Projekte. Deshalb gehören sie grundsätzlich in die Öffentlichkeit."

Im ersten Halbjahr 2011 übernimmt die Landesbeauftragte für Datenschutz und Informationsfreiheit der Freien Hansestadt Bremen den Vorsitz der Konferenz.

\section{Entschließungen}

Open Data: Mehr statt weniger Transparenz!

Die WikiLeaks-Debatte zeigt beispielhaft sowohl ein wachsendes Bedürfnis der in- ternationalen Öffentlichkeit nach verbesserter Information und mehr Transparenz staatlichen Handelns als auch nach einem wirksamen rechtsstaatlichen Rahmen für den Zugang zu öffentlichen Informationen. Auch in Deutschland muss die Transparenz des politischen Handelns einen deutlich höheren Stellenwert bekommen, indem die rechtlichen und tatsächlichen Möglichkeiten zum Zugang zu staatlichen Informationen verbessert werden. Die Informationsfreiheitsbeauftragten haben bereits vor vier Jahren die Verwaltungen aufgefordert, Informationen nicht erst auf Anfrage zu gewähren, sondern auch aus eigener Initiative im Internet $\mathrm{zu}$ veröffentlichen. Den Bürgerinnen und Bürgern soll damit der Zugang erleichtert und gleichzeitig der Aufwand für die öffentlichen Stellen mit der Bearbeitung von individuellen Anträgen auf Informationszugang reduziert werden.

Inzwischen ist einiges geschehen: Immer mehr Informationen, zum Beispiel über die Umwelt, Gerichtsentscheidungen, Parlamentsdokumente, amtliche Statistiken oder Vorlagen kommunaler Vertretungen, sind im Internet frei zugänglich. Aber immer noch fehlt ein Wegweiser durch die meist dezentral veröffentlichten Informationen ebenso wie ein einheitlicher technischer Standard, der die Weiterverwendung der Informationen erleichtern würde.

Beispiele aus dem In- und Ausland zeigen bereits heute, dass es möglich ist, eine Vielzahl von Informationen übersichtlich und über eine einheitliche Plattform zur Verfügung zu stellen.

So kann Transparenz gleichermaßen einen Beitrag zur Stärkung der Demokratie und auch zur effizienten Aufgabenwahrnehmung der Verwaltung leisten.

Verträge zwischen Staat und Unternehmen offen legen!

Öffentliche Stellen des Bundes, der Länder und der Kommunen bedienen sich bei der Wahrnehmung ihrer Aufgaben vielfach privater Unternehmen: von großen Firmen, die öffentliche Infrastrukturpro- jekte verwirklichen, bis hin zu kleinen Betrieben, die für eine Gemeinde das Dorffest arrangieren. Dabei nimmt der Umfang des Outsourcing ständig zu und umfasst auch zentrale Felder der staatlichen Daseinsvorsorge. Die wesentlichen Inhalte und Konditionen werden dabei vertraglich fixiert.

Das Interesse der Öffentlichkeit an den Inhalten solcher Verträge ist groß, die Bereitschaft der Vertragspartner, sie offen zu legen, meist gering. Bisweilen wird privaten Geschäftspartnern sogar die Vertraulichkeit der Vertragsbestimmungen ausdrücklich zugesichert, um deren Offenbarung zu vermeiden.

Von besonderem öffentlichem Interesse sind aussagekräftige Informationen über öffentliche Gelder, die für bestimmte Leistungen bezahlt wurden, ob die Leistungen mit den zuvor ausgeschriebenen Anforderungen übereinstimmen und in welcher Höhe Steuermittel dafür aufgewendet werden. Diese Angaben dienen der Haushaltstransparenz und der Verhinderung von Korruption. Transparenz bei derartigen Verträgen ist auch deshalb besonders wichtig, weil hier nicht selten langfristige Weichenstellungen getroffen werden, die auch Parlamente späterer Legislaturperioden nicht mehr ändern können. Angaben hierüber dürfen der politischen Diskussion nicht vorenthalten werden.

Die Informationsfreiheitsbeauftragten fordern deshalb, die Verträge zwischen Staat und Unternehmen grundsätzlich offen zu legen. Die pauschale Zurückweisung von auf solche Verträge gerichteten Auskunftsbegehren unter Hinweis auf Vertraulichkeitsabreden und Betriebsund Geschäftsgeheimnisse ist nicht länger hinnehmbar. Die Konferenz hält es deshalb für zwingend geboten, den Zugang zu entsprechenden Verträgen in den Informationsfreiheitsgesetzen sicherzustellen, wie dies jüngst im Berliner Informationsfreiheitsgesetz (GVBl. Berlin 2010, Seite 358) geschehen ist. 